\title{
Diabetes mellitus and Chronic Kidney Disease - Two Decades of Consultation of Diabetes and Kidney Transplant, a Retrospective Study
}

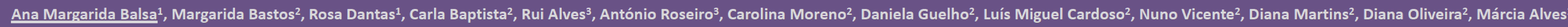
Joana Guimarães ${ }^{1}$, Alfredo Mota ${ }^{4}$ e Francisco Carrilho ${ }^{2}$

1-Serviço de Endocrinologia, Diabetes e Nutrição do Centro Hospitalar do Baixo Vouga, 2-Serviço de Endocrinologia, Diabetes e Metabolismo do Centro Hospitalar e Universitário de Coimbra, 3- Serviço de Nefrologia do Centro Hospitalar e Universitário de Coimbra 4-Serviço de Urologia e Transplantação Renal do Centro Hospitalar e Universitário de Coimbra

\section{Introduction}

Diabetic nephropathy is the main global cause of end stage renal disease. According to the annual report from the Portuguese National Diabetes Observatory, in 2013, the prevalence of Diabetes mellitus (DM) in new cases of chronic kidney disease was $31,2 \%$, and $11,1 \%$ in kidney transplant patients.

Potential recipients of a kidney transplant undergo a careful process of evaluation, selection and preparation.

\section{Goal}

To characterize the population of type 2 diabetic patients admitted in diabetes and kidney transplant consult (DKTC), comparing those admitted between the decade of Jan/1992Dec/2001 (D1) and Jan/2002-Dec/2011 (D2).

\section{Methods}

Retrospective analysis of type 2 diabetic patients admitted in DKTC between January 1992 and December 2011, registering the following data at first admission:

- age,

sex,

weight and $\mathrm{BMI}$,

age of diagnosis and DM duration,

treatment,

metabolic control,

- macro and microvascular complications.

Statistical analysis with SPSS ${ }^{\circledR}$.

\section{Resultados}

\section{Overall Characterization:}

238 patients (D1 $n=66, D 2 n=172$ ) of a total of 332 (D1 $\mathrm{n}=66$ e $\mathrm{D} 2 \mathrm{n}=266)$.

$58,28 \pm 7,737$ years

$15,76 \pm 8,689$ years of DM

- Comorbilidities:

Hypertension $-80,3 \%$

Dyslipidemia - 35,5\%

Oncologic Disease $-7,9 \%$
Figure 1. DM Treatment at $1^{\text {st }}$ admission

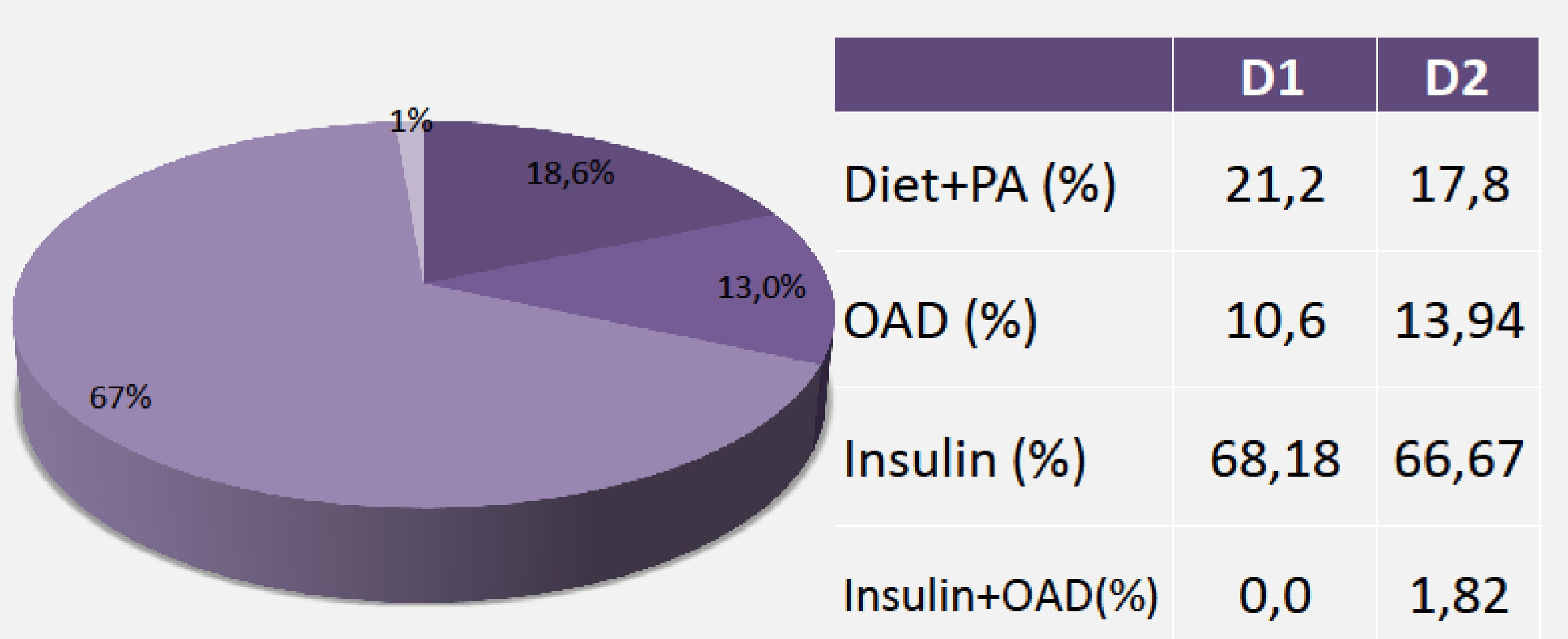

Fig 1: Diet+PA - diet and physical activity; OAD - Oral antidiabetic Drugs

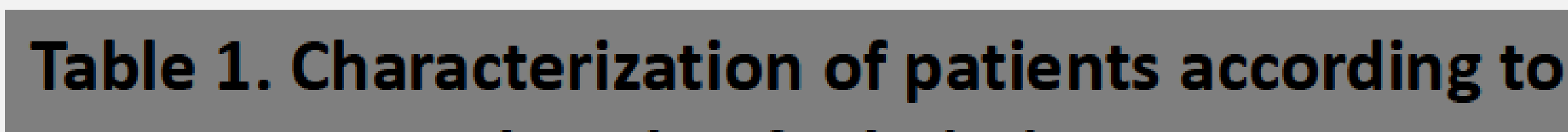
decade of admission

\begin{tabular}{|c|c|c|c|}
\hline & D1 & D2 & $\mathbf{p}$ \\
\hline № of patients & 66 & 172 & \\
\hline $\begin{array}{l}\text { Medium Age at } 1^{\text {st }} \\
\text { Admission (years) }\end{array}$ & $\begin{array}{l}56,91 \pm \\
7,75\end{array}$ & $\begin{array}{l}59,46 \pm \\
7,08\end{array}$ & 0,016 \\
\hline Duration of DM (years) & $\begin{array}{c}15,36 \pm \\
8,29\end{array}$ & $\begin{array}{c}15,76 \pm \\
8,61\end{array}$ & 0,750 \\
\hline $\begin{array}{l}\text { Medium BMI at } 1^{\text {st }} \\
\text { Admission }\left(\mathrm{Kg} / \mathrm{m}^{2}\right)\end{array}$ & $\begin{array}{c}27,25 \pm \\
0,552\end{array}$ & $\begin{array}{c}28,26 \pm \\
0,552\end{array}$ & 0,169 \\
\hline $\begin{array}{l}\text { Medium HbA1c at } 1^{\text {st }} \\
\text { Admission (\%) }\end{array}$ & $7,56 \pm 1,83$ & $7,13 \pm 1,47$ & 0,077 \\
\hline
\end{tabular}

Table 2. Frequency of macro or microvascular complications according to decade of admission (at $1^{\text {st }}$ admission)

\begin{tabular}{|c|c|c|c|}
\hline & D1 & D2 & $\mathbf{P}$ \\
\hline \multicolumn{4}{|c|}{ MICROVASCULAR COMPLICATIONS } \\
\hline Diabetic Retinopathy & $68,2 \%$ & $65,1 \%$ & 0,653 \\
\hline Proliferative Retinopathy & $51,5 \%$ & $43,1 \%$ & 0,246 \\
\hline Amaurosis & $7,6 \%$ & $10,7 \%$ & 0,476 \\
\hline Peripheric Neuropathy & $31,8 \%$ & $33,1 \%$ & 0,847 \\
\hline Amputation & $7,6 \%$ & $8,8 \%$ & 0,165 \\
\hline Major & $1,5 \%$ & $2,9 \%$ & 0,806 \\
\hline Minor & $6,1 \%$ & $5,9 \%$ & 0,293 \\
\hline $\begin{array}{l}\text { CORONARY ARTERY } \\
\text { DISEASE }\end{array}$ & $31,82 \%$ & $15,97 \%$ & 0,007 \\
\hline
\end{tabular}

\begin{tabular}{|c|c|c|c|}
\hline & D1 & D2 & Global \\
\hline \multicolumn{4}{|l|}{ Transplant } \\
\hline Total of transplanted patients & 22 & 53 & 75 \\
\hline $\begin{array}{l}\text { Patients with definitive } \\
\text { contraindication (\%) }\end{array}$ & 44,6 & 46,5 & 45,9 \\
\hline \multicolumn{4}{|l|}{ Orientation (\%) } \\
\hline $\begin{array}{l}\text { Discharge / Follow-up in } \\
\text { another institution }\end{array}$ & 43,94 & 34,40 & 37,70 \\
\hline Abandon & 31,82 & 16,80 & 21,99 \\
\hline Follow-up at CHUC & 24,24 & 48,8 & 40,3 \\
\hline
\end{tabular}

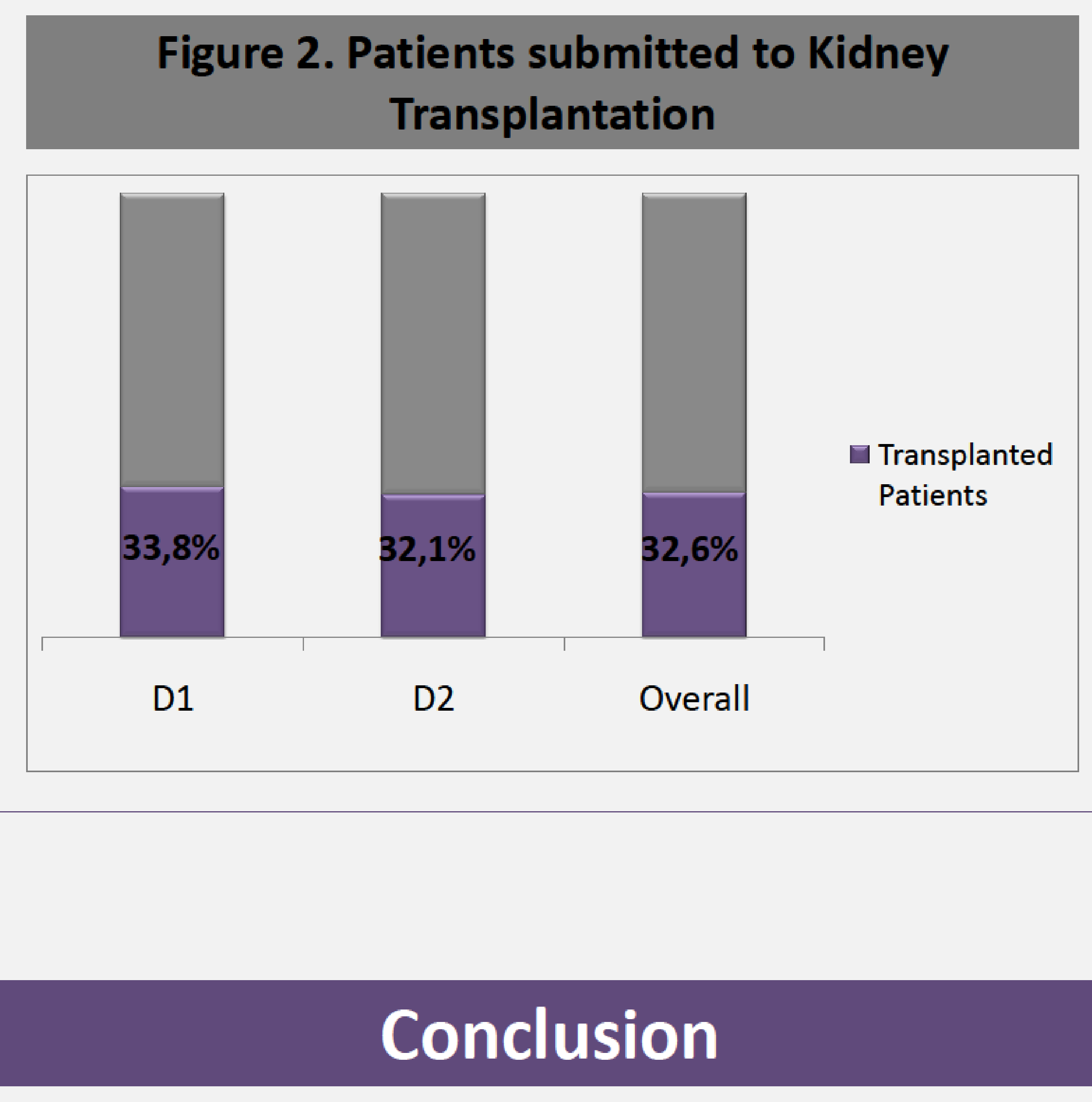

Diabetic patients admitted in DKTC generally have a long evolution of DM, with several comorbidities and complications.

Analyzing the first admission data from D1 and D2 patients only found statistical significant differences in patient's age (older at D2) and prevalence of coronary artery disease (larger at D1).

In the last decade, an increasing number of patients were referred to kidney transplantation, and although they were older, there was no larger prevalence of complications or comorbidities.

In two decades of follow-up only $32,6 \%$ of candidates were transplanted.

Because of their complexity theses patients need multidisciplinary and specific care.

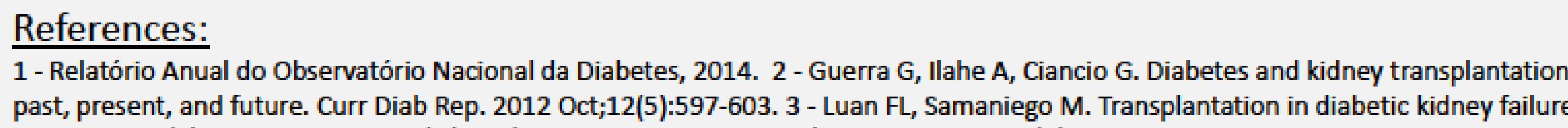

